\title{
Optimizing the 3D Microstructure Observation Conditions of Pearlite Steel through SEM-FIB Technique
}

\author{
Takashi Nishiyama, Haruo Nakamichi and Masayasu Nagoshi ${ }^{1}$ \\ Analysis \& Characterization Res. Dept., Steel Res. Lab., JFE Steel Corp., Kawasaki, Japan.
}

Microstructural information is very important for developing high grade steels. These days, the requirements for high-quality mechanical properties such as strength, elongation, toughness and so on are increasing for their use as construction materials. Along with the usual two-dimensional information, three-dimensional (3D) micro structural information is important for developing new materials. One method for evaluating 3D structure is serial sectioning imaging with using a combination of a scanning electron microscope (SEM) and a focused ion microscope (FIB) system[1]. This technique is powerful for obtaining tens of micrometer scales of 3D information and a reliable automated procedure has also been developed in the last couple of years. We installed SEM-FIB 3D analysis equipment for developing innovative automobile steel sheets through a Japanese national project for Innovative Structural Materials Development. From the viewpoint of industrial use, analysis in a reasonable time and with a reasonable resolution are required for automated 3D imaging. In the present paper, we try to optimize the 3D analysis conditions such as FIB fabrication and SEM observation conditions using a pearlite specimen. The effects of slice pitches are also evaluated because they have an effect on both analysis time and spatial resolution.

3D-SEM analysis was carried out using a Scios from FEI Corp., which has three through-lens detectors for T1, T2 and T3 - the so-called as Trinity lens system. Typical 3D microstructural analysis was carried out using pearlite steel with continuous FIB fabrication and SEM observations alternately. After obtaining a series of SEM images, 3D reconstructing was carried out using Avizo software(FEI).

Fig.1 shows SEM images of a FIB sliced plane observed with an E-T(Everhart-Thornley) detector, T1, $\mathrm{T} 2$ and T3 detectors with a $2 \mathrm{kV}$ accelerated voltage. It was found that good microstructural images can be obtained with using the T1 detector. On the other hand, the surface roughness which is formed through FIB slicing is observed by the T2 and T3 detectors. By comparison with other accelerated voltage observations, it is found that $2 \mathrm{kV}$ SEM observation using a T1 detector is optimized for observing the microstructure of a FIB sliced surface. Using the above condition ( $2 \mathrm{kV}$ accelerated with $\mathrm{T} 1)$, continuous SEM observations of the pearlite steel were carried out. In this experiment, FIB fabrication are carried out using an accelerated voltage of $30 \mathrm{kV}$ with slice pitches of $20 \mathrm{~nm}$ and a sliced volume of $25 \mathrm{x} 20 \mathrm{x} 3 \mu \mathrm{m}^{3}$. SEM observation were performed with $2 \mathrm{kV}$ accelerated voltage with using the T1 detector. Fig.2 shows the whole 3D image of the analysis area reconstructed with 150 SEM images. Lamella sheet distribution is clearly recognized and it was found that $\mathrm{T} 1$ detector observations have sufficient contrast to evaluate 3D images.

Using the above conditions, the 3D microstructure of pearlite lamella can be observed. However, discontinuous lamella are rarely observed and these lamella sheets tend to have a high angle orientation with the FIB slicing direction. To understand this matter, the effects of slice pitches on 3D reconstructions were evaluated. Fig. 3 shows 3D images of the same area where the slice pitches were changed to $20 \mathrm{~nm}$ and 100nm respectively. The100 nm slice pitch images were performed by reducing the number of SEM images from the original images of $20 \mathrm{~nm}$ pitches. It can be seen that the lamella sheet structure in the $20 \mathrm{~nm}$ case is continuous but that in the case of $100 \mathrm{~nm}$, the slice pitches become discontinuous. In 
evaluating the reason for this factors such as image quality, image drifting distance, target size, object area and so on with comparison of several images, it was found that the image overlapping had the most significant effect on 3D image constructing. An image overlapping of $1 / 3$ of the total is required between the serial images in order to obtain continuous 3D images with an automated procedure. In other words, a lamella sheet which is parallel to the FIB slice direction has less effect on slice pitches. However, the effects of slice pitches need to be considered if the lamella sheet has a high angle relation with the FIB slice direction. The sample used in this experiment has random orientation, but most of the structure can be seen through the 20nm slice pitched images. Slice pitches are directly connected with acquisition time and depth resolution so that this optimizing method is the most important factor for evaluating the structure.

This presentation is based on results obtained from a future pioneering project commissioned by the New Energy and Industrial Technology Development Organization(NEDO)

\section{References:}

[1] Y. Adachi et. al., Acta Materialia 56 (2008) 5995

[2] The authors acknowledge Mr. T. Kimura of JFE Steel for providing samples.
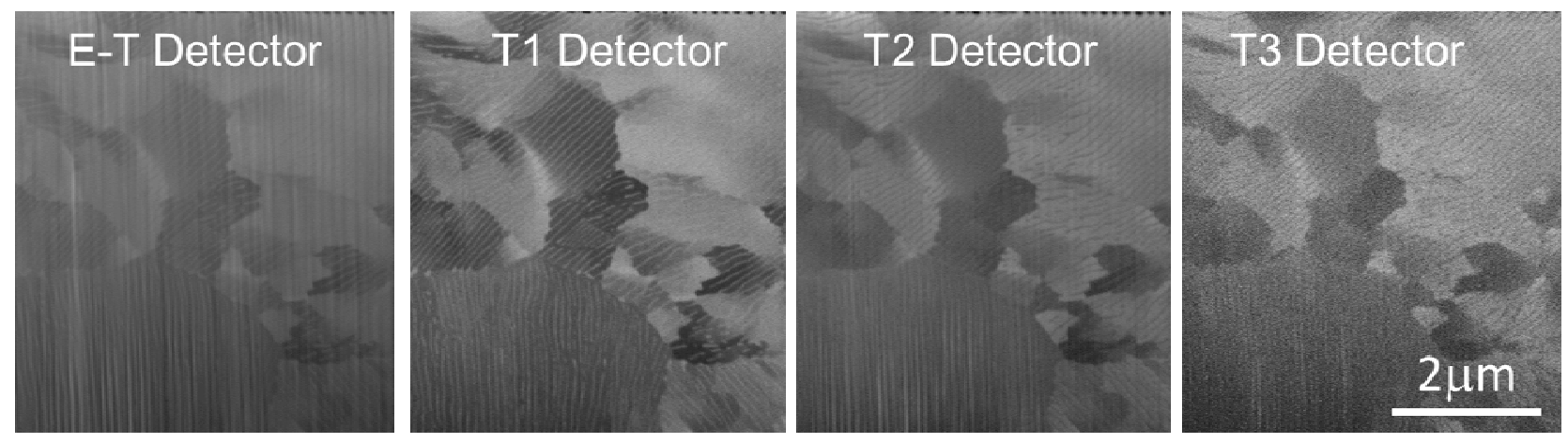

Figure 1. SEM images of FIB sliced cross sections of the same area taken concurrently by different detectors

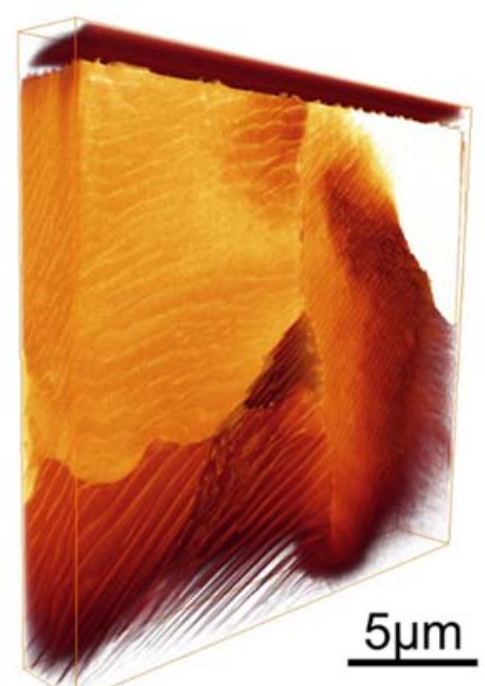

Figure 2. 3D image of pearlite steel
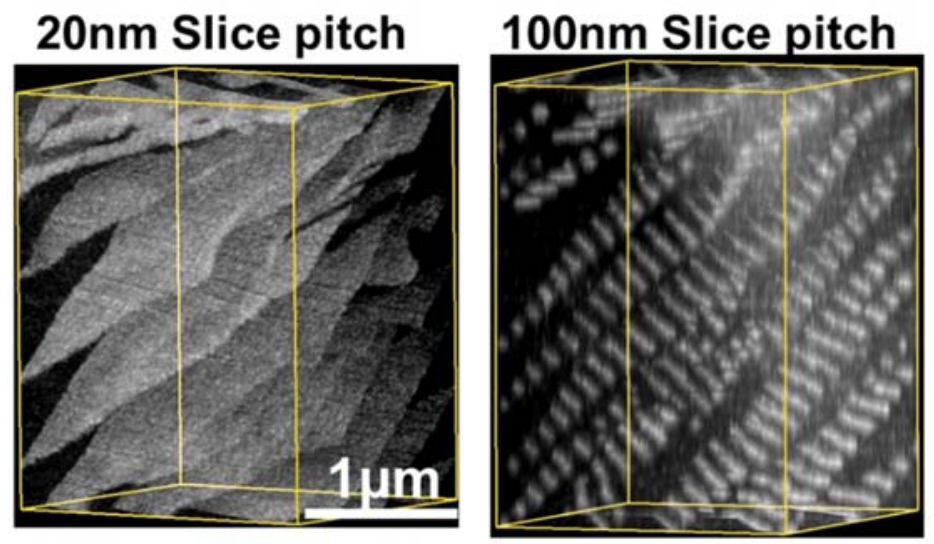

Figure 3. Slice pitch effects on 3D image 